\title{
Gains and losses in intertemporal preferences: a behavioural study
}

\author{
Valeria Faralla ${ }^{1}$, Francesca Benuzzi $^{2}$, Paolo Nichelli $^{2}$, Nicola Dimitri $^{3}$
}

\begin{abstract}
According to recent evidence (Frederick, Loewenstein, \& O'Donoghue, 2002), the traditional Discounted Utility model (Samuelson, 1937) has a limited ability to describe realistic models of behaviour and indeed there are several documented empirical regularities that seem to contradict this statement both in certainty and uncertainty conditions. This study focused on one of the best documented anomalies: sign effect or gain-loss asymmetry (Frederick et al., 2002; Loewenstein \& Prelec, 1992; Read, 2004). Specifically, the study investigated the intertemporal preference for symmetric monetary rewards and punishments in certain conditions, and the no wealth effects hypothesis (Dimitri, 2007) by asking subjects to choose between two positive or two negative euro amounts available at different points in time. The experimental design applied here followed the same behavioural pattern of the neuroeconomics' study on monetary rewards realized by McClure et al. (2004). The results confirmed a gain-loss asymmetry at least for medium and large euro amount and suggested new directions of research.
\end{abstract}

Keywords: intertemporal preferences; gains; losses; certainty; sign effect

J.E.L. classification: D90, D91

${ }^{1}$ Dipartimento di Filosofia e Scienze Sociali, Università di Siena, Italy

${ }^{2}$ Dipartimento di Neuroscienze, Università di Modena e Reggio Emilia, Italy

${ }^{3}$ Dipartimento di Politica Economica, Finanza e Sviluppo, Università di Siena, Italy

Very preliminary version: do not quote without the authors' permission 


\section{INTRODUCTION}

Time preference is a concept originally introduced by Rae (1834) in his study on differences of wealth among nations. According to Rae, time preference is determined by four different factors, which may positively or negatively affect the desire of accumulation: the bequest motive, the propensity to self-restraints, uncertainty of human life and the gratification derived from immediate consumption. Time preference was described as an intertemporal allocation of consumption by Fisher (1930) who enriched Rae's view with two additional personal factors: foresight and fashion. All these factors were later integrated into the discount rate of the Discounted Utility (DU) model (Samuelson, 1937). The DU model is based on various assumptions including stationary instantaneous utility, independence of discounting from consumption, diminishing marginal utility, positive time preference and, in particular, constant discounting which implies time-consistent preferences. Time consistency means that later preferences are in line with earlier preferences, namely preferences are constant across time. Precisely, individual time preferences are timeconsistent if "for any two consumption profiles $\left(c_{t}, \ldots, c_{T}\right)$ and $\left(c^{\prime}{ }_{t}, \ldots, c^{\prime}{ }_{T}\right)$, with $c_{t}=c^{\prime}$, $U^{t}\left(c_{t}, c_{t+1}, \ldots, c_{T}\right) \geq U^{t}\left(c^{\prime}{ }_{t}, c^{\prime}{ }_{t+1}, \ldots, c^{\prime}{ }_{T}\right)$ if and only if $U^{t+1}\left(c_{t}, c_{t+1}, \ldots, c_{T}\right) \geq U^{t+1}\left(c^{\prime}{ }_{t}, c^{\prime}{ }_{t+1}, \ldots, c^{\prime}{ }_{T}\right){ }^{\prime}$ (Frederick et al., 2002 p.358).

Although this model has been widely accepted and applied to the study of intertemporal choice, according to recent evidence (Frederick et al., 2002; Read, 2004), the classical DU model has a limited ability to describe realistic behaviour. Particularly, while DU assumes that the discount rate is the same for all time periods and different types of outcomes; there are several documented empirical regularities that seem to contradict this statement, both with certainty and uncertainty conditions. The present study focuses on sign effect or gain-loss asymmetry (Frederick et al., 2002; Loewenstein \& Prelec, 1992; Read, 2004) which implies that gains are discounted more than losses. Specifically, subjects usually prefer to expedite, rather than delay, negative outcomes as depicted by classical theory. It is not yet evident whether to consider the sign effect and other empirical regularities — such as magnitude effect, preference for improving sequences, or delay-speedup asymmetry - as subjects' errors in performing the task or, instead, as real anomalies of the classical DU model. In fact, in their study on workers' preferences for increasing wage profiles, Loewenstein and Sicherman write, "Respondents do not see themselves as violating any such fundamental property because they see no contradiction in deriving satisfaction from increasing payments, despite the effect on total consumption" (Loewenstein \& Sicherman, 1991). Nevertheless, this behavioural pattern is observed not only for monetary choices, but also for nonmonetary rewards and punishments. 


\section{LITERATURE REVIEW}

The empirical study of intertemporal rewards and punishments under certainty may be considered to have its roots in experimental psychology and, in particular, in the works by Mischel et al., originally conducted on both adults and children in the 60s. Particularly, in Mischel et al. (1969), effects of expected time delay on the subjective value of monetary and non-monetary rewards and punishments were investigated. The study presented the results obtained in four different experiments. In the first, children were asked to indicate their preferences for four different types of rewards - 50 cents, a notepad, six packs of chewing gum, 80 cents — and punishments — eat a can of bad tasting food, listen to a noise for 15 minutes, spend 30 minutes with an unpleasant teacher, or do four difficult tasks in front of the class - available immediately or after a delay of one day, one week, or three weeks. Data from this first experiment showed that subjective value of children for positive outcomes decreased with time delay and also that immediate rewards were preferred to delayed ones, at all delay levels. Nevertheless, this was not true for immediate punishments and, indeed, immediate negative outcomes were neither more nor less preferred as delayed ones. These results were confirmed in the second experiment in which the two alternatives were both delayed (immediate options were no longer available). The third experiment of the study was conducted on adults. Although the researchers applied similar experimental procedures, rewards and punishments were slightly modified in order to be more suitable for older subjects. Data were consistent with the children's findings for positive outcomes. For losses, instead, adults preferred immediate punishments to delayed ones, indicating a preference for anticipating losses. Since the effect was not so significant, however, Mischel et al. carried out a final experiment in which they asked participants — adults - choose between different electric shocks. In particular, the electric shock was higher in voltage and time duration for one group and lower for a second group. These differences were applied in order to investigate the relative importance of punishment intensity. As a result, Mischel et al. found that the stronger shocks were less preferred. Moreover, subjects tended to choose the more immediate shock irrespectively of the intensity and the duration, confirming previous results. Note that Mischel et al. also found no correlation between the state of anxiety and the results obtained in this fourth experiment. The general findings by Mischel et al. were consistent with experimental research on children (Grusec, 1968), adults (Hare, Krebs, Creighton, \& Petrusic, 1966) and with shorter time delays (Cook \& Barnes, 1964; Hare, 1966).

The work by Mischel et al. inspired a subsequent experiment on monetary losses, realized by the psychologists Yates and Watts (1975). In their study, participants had to choose between two monetary options that they would prefer to pay. Each binary option was represented by a pie and was different in monetary amount to be repaid ( $\$ 1$ and $-\$ 2)$ and time delay $(15,35$, or 75 days). 
They also introduced an element of uncertainty which was indicated by a slight $(\mathrm{p}=0.6)$ or complete $(\mathrm{p}=1)$ darkening of the pie. Binary choice with identical net amounts was also presented under controlled conditions in which other participants were invited to select one between two positive monetary options. Yates and Watts classified their subjects as "deferrers" or "advancers". Deferrers tended to procrastinate the outcome, whereas advancers generally preferred the more immediate option. As a result, they found that the number of advancers and deferrers under the two conditions was completely different. In particular, in the experimental condition - negative amounts, they found an equal number of advancers and deferrers (8 and 8) while in the control condition positive amounts - they found a large prevalence of advancers (18 out of 19). Since the two groups obtained comparable scores in the anxiety test, this was not due to a possible state of anxiety. Given these facts, their data analysis supported the hypothesis that people were typically agreed to expedite monetary gains but not losses as in Mischel et al. (1969). Yates and Watts attributed this kind of result to the possibility that Mischel et al. were unsuccessful in separating, in the subject's perception, compensation for participation and real outcome for preferred choice. As a matter of fact, punishments were not perceived as real negative outcomes by subjects.

As far economists, some years later, Thaler (1981) continued to investigate the issue of gains and losses in intertemporal choice in the framework of dynamic consistency (Strotz, 1955-56). In his study, participants were asked to complete four different forms. For each form three immediate monetary amounts were given: $\$ 15, \$ 250$, or $\$ 3000$, in the first; $\$ 75, \$ 250$, or $\$ 1200$, in the second; and $\$ 15, \$ 250$, or $\$ 3000$, in the third. Different time delays for these three euro amounts were given: 3 months, 1 year, or 3 years, in the first form; 6 months, 1 year, and 5 years, in the second form; 1 month, 1 year, and 10 years, in the third form. The fourth form was characterized instead by three negative dollar amounts: $-\$ 15,-\$ 100$, and $-\$ 250$. Time delays were, in this case, 3 months, 1 year, and 3 years. Data revealed very high implicit discount rates, negatively correlated with both size of the outcome, for gains and losses, and time delay, for gains only. Most important, these rates were smaller for losses than for gains. Compared with the previous studies of Mischel et al. and Yates and Watts, Thaler introduced longer time delays - years instead of days. Nonetheless, he also provided evidence for sign effect as Mischel et al., confirming that intertemporal preferences for gains and losses are not the same.

Later, Loewenstein (1988) and Benzion et al. (1989) applied Thaler's method of implicit discount rates in other experiments on intertemporal preferences for monetary gains and losses, obtaining similar results. In particular, Loewenstein (1988) investigated the frame effect in intertemporal choice of monetary rewards and punishments; that is, how different questionnaire formats 
influenced the weighting of waiting and, in general, intertemporal choice (the connections between question frame and discount rates for gains and losses have been also investigated by Shelley, 1993). Loewenstein also found higher discount rates for gains than for losses, confirming previous findings by Thaler (1981). Loewenstein suggested that waiting for a punishment is generally unpleasant. Consequently, waiting for a negative outcome introduces an additional cost which could be responsible for lower discount rates. In contrast, waiting for a reward could be pleasant or frustrating depending on the outcome. In particular, waiting is frustrating for people with impulsivity and self-control problems. This is especially true when the delay is initially unexpected or when both the amount of the outcome and the time delay are high.

Benzion et al. (1989) proposed a questionnaire to participants with items from four different scenarios: postpone a receipt, postpone a payment, expedite a receipt, and expedite a payment. As a matter of fact, one could consider scenarios one and three as gain frames, two and four as loss frames. Data analysis confirmed the previous results of Thaler (1981). Benzion et al. found, in fact, that implicit discount rates were decreasing as time delay and monetary amount increased and, in particular, such rates were smaller for losses (scenarios two and fourth) than for gains (scenarios one and three). Although the findings of Benzion et al. supported the hypothesis of sign effect. Implicit discount rates were smaller than in Thaler and, moreover, differed widely across participants. Benzion et al. underlined that this last result could be determined by inter- and intraindividual differences in economic knowledge or approach regarding the future. Note that in this experiment the immediate option was not available and, in fact, time delays were 6 months (which is the more immediate option), 1, 2, or 4 years.

As noted earlier, gain-loss asymmetry has been investigated not only for monetary outcomes but also for non-monetary rewards and punishments. In particular, Loewenstein (1987) reported the results of a study in which participants were asked to indicate the amount that they would pay immediately to have $\$ 4$ or a kiss from their favourite movie star, for gains, and avoiding to lose $-\$ 4$, $-\$ 1000$, or receiving an electric shock, for punishments. These rewards and punishments were immediately available (three hours) or could be delayed for 24 hours, 3 days, 1, or 10 years. Data revealed that implicit discount rates were higher for non-monetary rather than for monetary items. Moreover, subjects were willing to pay more for a non-monetary reward - the kiss - which was not immediately available and for avoiding a non-monetary punishment — the electric shock — which was delivered with long time delays, that is one or three days.

Preference for anticipating aversive events was also demonstrated to be a very robust preference pattern by Prelec and Loewenstein (1998). In their study, Prelec and Loewenstein presented a 
"double-entry mental accounting model" which took into account both the pleasure of consumption and the pain of paying, which could adversely affect consumption itself considering the payment as a potential loss. To test the model, Prelec and Loewenstein conducted an experiment in which participants were asked to plan a vacation in a luxury apartment. Considering that the apartment cost them $\$ 3000$ for three weeks, participants were also required to rate different hypothetical situations. Indeed, different combinations for vacation time and payment for consumption were given. As a result, Prelec and Loewenstein found that the majority of people actually preferred prepayment or, at least, to alternate consumption with payment for the vacation. In any case, however, participants mainly avoided situations in which the vacation payment was entirely delayed. These findings not only refuted the statement that the discount rate should be constant for all time periods and different types of outcomes but also confirmed the hypothesis that people prefer to expedite payments, which was in line with previous studies with monetary and nonmonetary punishments, as previously mentioned. This particular pattern of choice could be connected with the need to be free of payment concerns - during or after consumption — and likely derived from a strong debt aversion.

Based on the state of the art found in the literature, the present study analyzed individual behaviour in choosing symmetric monetary rewards and punishments under certain conditions and the no wealth effects hypothesis (Dimitri, 2007) in order, primarily, to provide test evidence for sign effect or other empirical anomalies (Frederick et al., 2002). Moreover, a statistical analysis was realized in order to identify significant variables in the choice between short and long-term positive and negative outcomes: euro amount, time delay, and percent difference between the two alternatives of choice. Gender and schooling effects were also evaluated.

\section{METHODS}

\section{Participants}

Twenty-five students were recruited to participate in the study, including 13 females and 12 males, with mean age 25.5 years and standard deviation 5. Each subject took about 21 minutes to complete the task (s. d. 7).

\section{Materials and procedures}

Before the experiment, subjects were told that they were participating in a study concerning the theory of cognitive processes in decision making. They were instructed that there were no correct answers, but that they were required to state their preferences in a series of positive and negative 
choice tasks. Subjects were informed that at the end of the experiment they would be paid $€ 5$ in cash as a participation fee and told they could receive an additional reward which would be paid in the form of a top-up mobile telephone. Specifically, the final reward given to each subject would be determined by random extracting - the uniform distribution was applied — one of the positive and negative choices they made. The choices selected were added and approximated to the nearer topup mobile telephone's size for a minimum of $€ 8$ and a maximum of $€ 15$. The top-up would be made on the day calculated as the mean between the time delays of the selected preferences. Given this, subjects were explicitly told that because of this payment scheme they would make choices as if it were the one they were actually going to receive. This method of reward was thought of in order to tie the monetary incentive to the performance task.

Stimulus presentation and behavioural response collection were controlled by the software Superlab (4.0, Cedrus Corporation, Inc.). Stimuli were adapted from the experimental paradigm used by McClure et al. (2004) on immediate and delayed monetary rewards. The first two questions in the task were fixed to allow subjects to get acclimated to task performance and acquire familiarity with the experimental tools. Each subject completed a task comprising 240 positive and negative monetary choices (120 gains and 120 losses) displayed as a pair of sentences. Choices were generated by combining three different variables: size of the outcome, time delay and percent difference. Three different smaller, earlier euro amounts were used as possible outcomes $(€ A)$ : small (€5), medium (€15), and large (€30) for gains; symmetric small (-€5), medium (-€15), and large $(-€ 30)$ for losses. For both gains and losses, there were five possible time delays between the late and the early euro amount: today-2weeks, today-1month, 2weeks-1month, 2weeks1 monthand2weeks, and 1month-1monthand2weeks. The percent differences in Euros between the two alternative of choice $\left[\left(€ A^{\prime}-€ A\right) / € A\right]$ were $1 \%, 3 \%, 5 \%, 10 \%, 15 \%, 25 \%, 35 \%$, or $50 \%$. The larger amount $\left(€ A^{\prime}\right)$ was set so that $\left[\left(€ A^{\prime}-€ A\right) / € A\right]$ was the desired percent difference. The smaller, earlier gain/loss was always presented on the left side of the computer screen and the sequence of choices were randomly generated by Superlab. The choice between the early and the late amount was always for the same type of monetary outcome (gain or loss), and each stimulus was presented only once.

Each experiment was conducted in only one session. Subjects were sitting in front of a computer screen on which the task was presented. For each choice, subjects were asked to select the key A or $\mathrm{L}$ on the keyboard for respectively selecting the smaller, earlier alternative or the larger, later outcome. No time limits were given for the experiment. Once the subject made the choice, the screen remained white for 500 milliseconds to signal the presentation of the new stimulus. Each 
choice was made with the event editor of Superlab using black Geneva $72 \mathrm{pt}$ fonts on white screen colour $(\mathrm{R}=250 ; \mathrm{G}=250 ; \mathrm{B}=250)$ (Figure 1).

Data were analyzed using software R (2.7.2, R Foundation for Statistical Computing, Inc.).

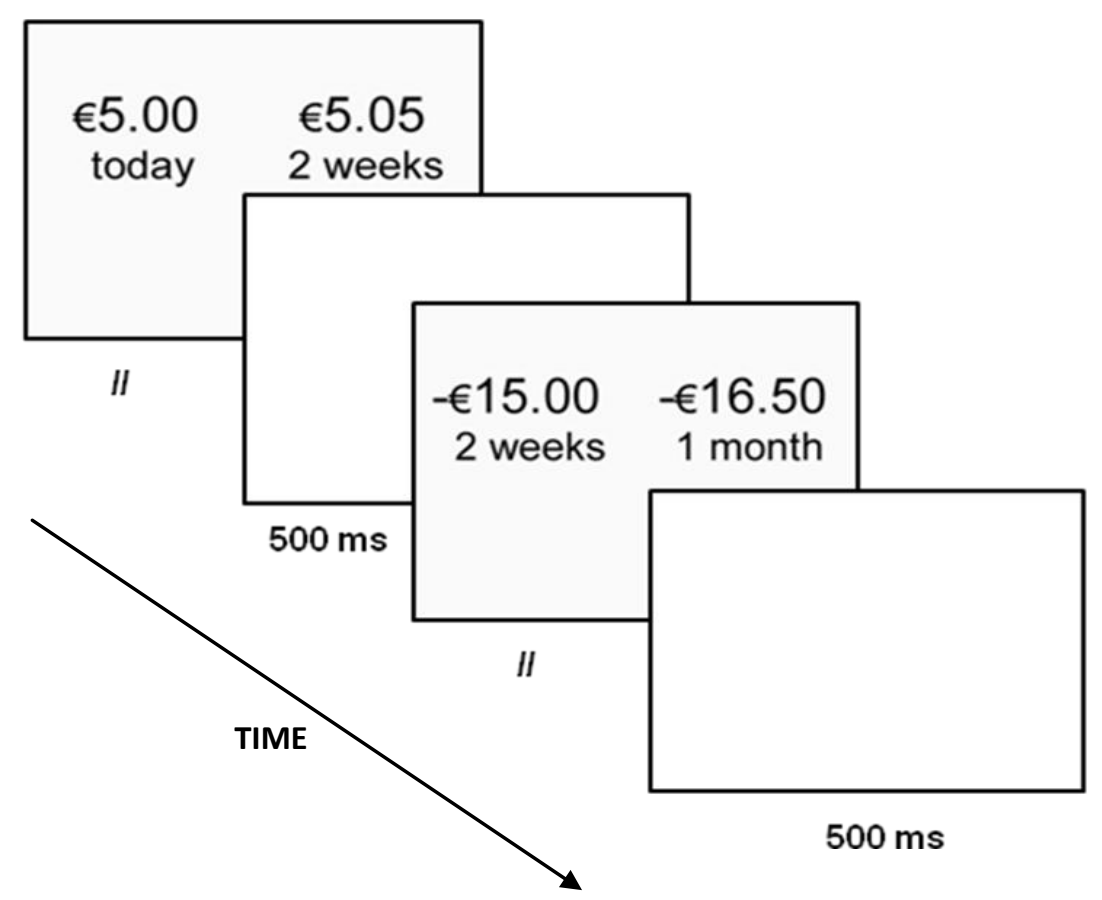

Figure 1. Temporal sequence of events during trials of the experimental session (gain and loss, respectively)

\section{RESULTS}

Descriptive analysis of the data revealed that subjects preferred the smaller, earlier alternative for gains $(61.50 \%)$ with a percentage that was decreasing with the value of the euro amount. For losses, instead, even if there was not a clear pattern of preference for one of the two options - and indeed the difference between the preference for the smaller, earlier loss and the larger, later one was of only $4.8 \%$ (Figure 2 ); outcomes were typically delayed for small outcomes (-€5) but expedited for intermediate and large ones (-€15 and -€30). Considering gender, moreover, males showed a little more preference for the larger, later option for losses $(52.56 \%)$ and smaller, earlier one for gains $(60.96 \%)$; whereas females tended to choose the smaller, earlier alternative for both losses and gains $(57.78 \%$ and $62.08 \%$, respectively), yielding results in the opposite direction. These results were reasonably consistent between both graduates and undergraduates, even if the first group seemed to be more undecided than the second one between smaller (earlier) and larger (later) alternatives. 

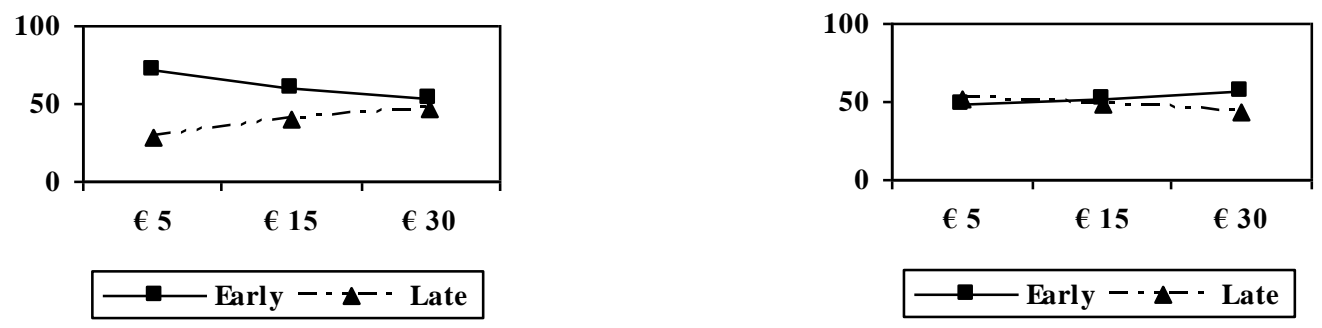

Figure 2. Percentage of responses for gains (on the left) and losses (on the right)

Analysis of the variable time delay revealed that preferences were rather swinging and this is especially true for losses. From the point of view of percent difference between the two alternatives of choice, instead, it was possible to discern a particular pattern of choice. Indeed, the percentage of responses for early losses was increasing with the percent difference between the smaller, earlier and the larger, later outcome (Figure 3). The higher increment was observed between 5\% and 10\% $(13.33 \%)$ and $15 \%$ and $25 \%(13.60 \%)$. Inversely, considering choices that involved gains, the pattern of choice was decreasing. Nevertheless, the higher percentage of responses continued to be between $5 \%$ and $10 \%(-16 \%)$. As a matter of fact, subjects expedited gains and delayed losses when the percent difference between the two alternatives was low (up to 5\%). In contrast, they delayed gains and expedited losses when the percentage was large (from $25 \%$ to 50\%). For intermediate percent differences $(10 \%$ and $15 \%)$, subjects preferred the early alternative irrespective of the sign of the payoff. This findings were consistent for both gender and schooling.
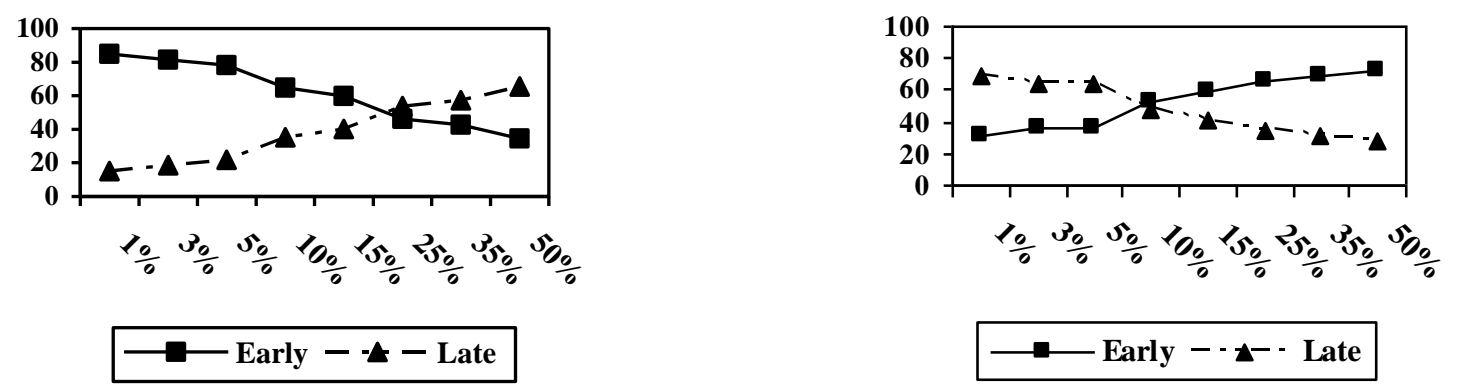

Figure 3. Percentage of responses for gains (on the left) and losses (on the right) for percent difference between the smaller, earlier and the larger, later outcome

For completeness and accuracy, data were also analyzed with the analysis of variance (ANOVA) for multiple regression analysis. Because of the complexity of the experimental paradigm, however, the significance of the variables was also valued with logistic-normal model for binary data (Agresti, 2002), obtaining quite similar results. As a result, for positive choices, the analysis yielded a significant effect on different variables: euro amount $(\mathrm{p}<0.001)$, time delay $(\mathrm{p}<0.001)$, percent difference $(p<0.001)$, schooling $(p<0.01)$, and age $(p<0.001)$. The analysis completed for negative 
choices yielded a significant effect for all variables: euro amount $(\mathrm{p}<0.001)$, time delay $(\mathrm{p}<0.001)$, percent difference $(\mathrm{p}<0.001)$, gender $(\mathrm{p}<0.001)$, schooling $(\mathrm{p}<0.05)$, and age $(\mathrm{p}<0.001)$. Combining positive and negative choices, the analysis yielded significant effect for euro amount $(\mathrm{p}<0.001)$, gender $(\mathrm{p}<0.001)$, and schooling $(\mathrm{p}<0.001)$.

\section{Matching analysis}

The data from the study were also modelled and interpreted by matching subjects' answers for symmetric gains and losses. So, we can refer to this additional behavioural result as matching analysis. For simplicity, a specific code was assigned to the possible combinations of matching: code 0 if the subject preferred the earlier alternative for symmetric gain and loss; code 1 if the subject preferred the later alternative for symmetric gain and loss; code 2 if the subject preferred the earlier gain but the later symmetric loss; code 3 if the subjects preferred the later gain but the earlier symmetric loss. In accordance with the pattern of choice, code 0 and code 1 were defined as concordant; whereas, code 2 and code 3 were discordant. The aim of the matching analysis was to verify if subjects were time-consistent. As noted earlier, time consistency means that later preferences are in line with earlier preferences, namely preferences are constant across time. Considering both gains and losses, in particular, a person is dynamic consistent if she prefers a larger, later gain and a smaller, earlier loss or vice versa (Dimitri, 2007). As a result, only codes 2 and 3 could be considered as time-consistent. Matching results for the behavioural study are summarized in Table 1 and Figure 4.

\begin{tabular}{lrr}
\hline Code & Total & $\begin{array}{r}\text { Total } \\
(\boldsymbol{\%})\end{array}$ \\
\hline $\mathbf{0}$ & 730 & 24.33 \\
$\mathbf{1}$ & 313 & 10.43 \\
$\mathbf{2}$ & 1115 & 37.17 \\
$\mathbf{3}$ & 842 & 28.07 \\
Total & $\mathbf{3 0 0 0}$ & $\mathbf{1 0 0}$ \\
\hline
\end{tabular}

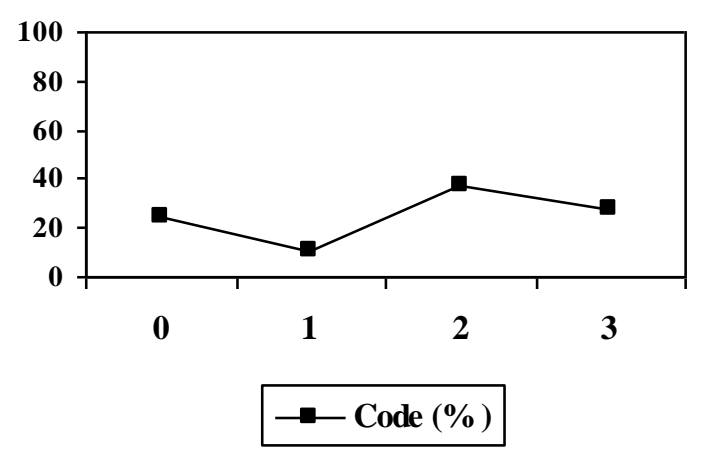

Table 1. Matching results (number and percentage of responses) Figure 4. Percentage of responses for matching As Table 1 shows, code 2 was the most preferred with a percentage of $37.17 \%$, followed by code 3 $(28.07 \%)$, code $0(24.33 \%)$, and code 1 (10.43\%). As a matter of fact, subjects preferred the smaller, earlier gain but the larger, later symmetric loss, even if the gap between the two most preferred codes was not really large $(9.1 \%)$. The pattern of choice described by code 2 continued to be consistent across all the variables investigated (euro amount, time delay, and percent difference 
between earlier and later outcomes); even if, code 3 was chosen when the euro amount and the percent difference between the smaller, earlier and the larger, later outcome were high ( $€ 30$ and $25 \%, 35 \%$, or $50 \%$ ) or when the time delay was long (1month-1monthand2weeks). Note that although they cannot be considered as consistent intertemporal preferences, the percentage of responses for discordant codes, especially code 0, was high. In reference to the matching analysis, males and females were quite different in their preferences according to their level of education. Specifically, with higher levels of schooling, females tended to move from code 3 (30.93\%) — and 0 (30.97\%), to code 2 (38.06). Inversely, males increased their capacity of long-term planning moving from code $2(45.28 \%)$ to $3(35.63 \%)$; even if the percentage assigned to code 3 was not far from code $2(35.21 \%)$. We have to notice that the female pattern did not seem to be influenced by instinct as suggested by reaction time. These switching effects are displayed in Table 2.

\begin{tabular}{lrrrrrr}
\hline & \multicolumn{2}{c}{ Undergraduates } & \multicolumn{2}{c}{ Graduates } & \\
\cline { 2 - 5 } Code & Males & Females & Males & Females & $\begin{array}{r}\text { Total } \\
(\%)\end{array}$ \\
\hline $\mathbf{0}(\%)$ & 20.74 & 30.37 & 14.38 & 30.28 & $\mathbf{2 4 . 3 3}$ \\
$\mathbf{1}(\%)$ & 8.43 & 9.07 & 14.79 & 14.72 & $\mathbf{1 0 . 4 3}$ \\
$\mathbf{2}(\%)$ & 45.28 & 29.63 & 35.21 & 38.06 & $\mathbf{3 7 . 1 7}$ \\
$\mathbf{3}(\%)$ & 25.56 & 30.93 & 35.63 & 16.94 & $\mathbf{2 8 . 0 7}$ \\
Total $(\boldsymbol{\%})$ & $\mathbf{1 0 0}$ & $\mathbf{1 0 0}$ & $\mathbf{1 0 0}$ & $\mathbf{1 0 0}$ & $\mathbf{1 0 0}$ \\
\hline
\end{tabular}

Table 2. Matching by sex and schooling (percentage of responses)

The analysis of variance yielded a significant effect for euro amount $(\mathrm{p}<0.001)$, percent difference $(\mathrm{p}<0.05)$, schooling $(\mathrm{p}<0.01)$, and gender $(\mathrm{p}<0.001)$, for gains, and euro amount $(\mathrm{p}<0.001)$, time delay $(\mathrm{p}<0.001)$, percent difference $(\mathrm{p}<0.001)$, gender $(\mathrm{p}<0.01)$, and age $(\mathrm{p}<0.001)$, for losses.

\section{DISCUSSION AND CONCLUSIONS}

The aim of this study was to investigate individual behaviour in choosing symmetric monetary rewards and punishments under certainty. We also evaluated the effect of monetary amount, time delay, percent difference, gender, and schooling. As in previous research, results showed that gains and losses are not equal and seem to be drawn by different internal principles of choice. First of all, according to previous findings (Frederick et al., 2002), evidence for gain-loss asymmetry was found, although only for intermediate and large euro amounts. Specifically, despite there was not a distinct pattern of preference for one of the two given losses at a general level, subjects really expedite negative outcomes when the loss was intermediate or large ( $€ 15$ and $-€ 30)$. Instead, for gains, we found a preference for earlier, smaller alternatives, independently of the size of the 
outcome. Note that in previous studies (Bickel \& Madden, 1999; Kirby, Petry, \& Bickel, 1999; Madden, Petry, Badger, \& Bickel, 1997) people who preferred short-term rewards were found to show impulse and addictive behaviours and lack of self-control. Secondly, considering the percent difference between short and long-term alternatives of choice, the percentage of responses for early outcomes was increasing for losses and decreasing for gains, as the percent difference variable raised. In fact, subjects expedited gains and delayed losses when the percent difference between the two alternatives was low (up to 5\%) but, inversely, they delayed gains and expedited losses when this percentage was large (from $25 \%$ to $50 \%$ ). As a result, the present findings also suggested that magnitude effect, another empirical regularity which contradicts the classical DU model, played here a specific role in intertemporal choice, at least for losses. Thirdly, preferences among different time delays were swinging, especially for losses; therefore, we could not identify a particular pattern of choice. Fourthly, switching effects were revealed for both males and females with increasing level of education and, indeed, females reversed their choices from code 3 to code 2, whereas males moved precisely in the opposite direction (from code 2 to 3 ). Note that gender differences in preferences were also found in Croson and Gneezy (2009). Finally, according to the statistical analysis, we found significant p-value especially for euro amount, gender and schooling.

As stated in the literature reviewed, sign effect is not new in empirical research and indeed gain-loss asymmetry was found both for monetary and non-monetary rewards and punishments. Particularly, most previous studies found that gains are discounted more than losses (Benzion et al., 1989; Loewenstein, 1988; Thaler, 1981) and non-monetary punishments have been found to be preferred sooner than later (Loewenstein, 1987; Mischel et al., 1969). The question of sign effect, however, is still open and indeed there are also other empirical studies highlighting that people do not overwhelmingly prefer to anticipate punishments (Yates \& Watts, 1975), which is also summarized in Read (2004).

In light on these findings and for better understanding gain-loss asymmetry, we suggest to guide further researches on gains and losses in intertemporal preferences towards new empirical directions as the result of a multiple-motive approach. The paper by McClure et al. (2004) represented an important step in the neuroeconomics of intertemporal choice; however, their study considered only monetary rewards. Now, it could be of interest to realize additional studies with both positive and negative alternatives of choice (Xu, Liang, Wang, Li, \& Jiang, 2009). This kind of study could verify the possibility of different neural circuits in the processing of immediate and delayed losses as well, and it can also verify if these activations are the same as those that emerged for gains. A similar project could contribute to cognitive economics, considering intertemporal preferences with 
more detail, and to neuroscience by deepening the interaction among neural systems. This new direction is obviously filled with critical aspects but thanks to neuroscience tools it is now possible to proceed in this worthwhile direction.

\section{Acknowledgments}

The authors thank Alessandro Innocenti (Dip. Politica Economica, Finanza e Sviluppo, Univ. di Siena) and Francesco Fallucchi (University of Texas at Austin, USA) for their valuable help. A special thank goes also to Lucio Barabesi and Luigi Greco (Dip. Metodi Quantitativi, Univ. di Siena), Andrea Cerioli (Dip. di Economia, Univ. di Parma), and Arsenio Stabile (Dip. Politica Economica, Finanza e Sviluppo, Univ. di Siena) for statistical advice.

\section{APPENDIX. Supplementary data}

\section{Table 1. Stimuli list}

\begin{tabular}{|c|c|}
\hline Gains & $\underline{\text { Losses }}$ \\
\hline 1. $€ 5.00$ today vs. $€ 5.052$ weeks & 1. $-€ 5.00$ today vs. $-€ 5.052$ weeks \\
\hline 2. $€ 5.00$ today vs. $€ 5.051$ month & 2. $-€ 5.00$ today vs. $-€ 5.051$ month \\
\hline 3. $€ 5.002$ weeks vs. $€ 5.051$ month & 3. $-€ 5.002$ weeks vs. $-€ 5.051$ month \\
\hline 4. $€ 5.002$ weeks vs. $€ 5.051$ month and 2 weeks & 4. $-€ 5.002$ weeks vs. $-€ 5.051$ month and 2 weeks \\
\hline 5. $€ 5.001$ month vs. $€ 5.051$ month and 2 weeks & 5. $-€ 5.001$ month vs. $-€ 5.051$ month and 2 weeks \\
\hline 6. $€ 15.00$ today vs. $€ 15.152$ weeks & 6. $-€ 15.00$ today vs. $-€ 15.152$ weeks \\
\hline 7. $€ 15.00$ today vs. $€ 15.151$ month & 7. $-€ 15.00$ today vs. $-€ 15.151$ month \\
\hline 8. $€ 15.002$ weeks vs. $€ 15.151$ month & 8. $-€ 15.002$ weeks vs. $-€ 15.151$ month \\
\hline 9. $€ 15.002$ weeks vs. $€ 15.151$ month and 2 weeks & 9. $-€ 15.002$ weeks vs. $-€ 15.151$ month and 2 weeks \\
\hline 10. $€ 15.001$ month vs. $€ 15.151$ month and 2 weeks & 10. $-€ 15.001$ month vs. $-€ 15.151$ month and 2 weeks \\
\hline 11. $€ 30.00$ today vs. $€ 30.302$ weeks & 11. $-€ 30.00$ today vs. $-€ 30.302$ weeks \\
\hline 12. $€ 30.00$ today vs. $€ 30.301$ month & 12. $-€ 30.00$ today vs. $-€ 30.301$ month \\
\hline 13. $€ 30.002$ weeks vs. $€ 30.301$ month & 13. $-€ 30.002$ weeks vs. $-€ 30.301$ month \\
\hline 14. $€ 30.002$ weeks vs. $€ 30.301$ month and 2 weeks & 14. $-€ 30.002$ weeks vs. $-€ 30.301$ month and 2 weeks \\
\hline 15. $€ 30.001$ month vs. $€ 30.301$ month and 2 weeks & 15. $-€ 30.001$ month vs. $-€ 30.301$ month and 2 weeks \\
\hline 16. $€ 5.00$ today vs. $€ 5.152$ weeks & 16. $-€ 5.00$ today vs. $-€ 5.152$ weeks \\
\hline 17. $€ 5.00$ today vs. $€ 5.151$ month & 17. $-€ 5.00$ today vs. $-€ 5.151$ month \\
\hline 18. $€ 5.002$ weeks vs. $€ 5.151$ month & 18. $-€ 5.002$ weeks vs. $-€ 5.151$ month \\
\hline 19. $€ 5.002$ weeks vs. $€ 5.151$ month and 2 weeks & 19. $-€ 5.002$ weeks vs. $-€ 5.151$ month and 2 weeks \\
\hline
\end{tabular}




\begin{tabular}{|c|c|}
\hline 20. $€ 5.001$ month vs. $€ 5.151$ month and 2 weeks & 20. $-€ 5.001$ month vs. $-€ 5.151$ month and 2 weeks \\
\hline 21. $€ 15.00$ today vs. $€ 15.452$ weeks & 21. $-€ 15.00$ today vs. $-€ 15.452$ weeks \\
\hline 22. $€ 15.00$ today vs. $€ 15.451$ month & 22. $-€ 15.00$ today vs. $-€ 15.451$ month \\
\hline 23. $€ 15.002$ weeks vs. $€ 15.451$ month & 23. $-€ 15.002$ weeks vs. $-€ 15.451$ month \\
\hline 24. $€ 15.002$ weeks vs. $€ 15.451$ month and 2 weeks & 24. $-€ 15.002$ weeks vs. $-€ 15.451$ month and 2 weeks \\
\hline 25. $€ 15.001$ month vs. $€ 15.451$ month and 2 weeks & 25. $-€ 15.001$ month vs. $-€ 15.451$ month and 2 weeks \\
\hline 26. $€ 30.00$ today vs. $€ 30.902$ weeks & 26. $-€ 30.00$ today vs. $-€ 30.902$ weeks \\
\hline 27. $€ 30.00$ today vs. $€ 30.901$ month & 27. $-€ 30.00$ today vs. $-€ 30.901$ month \\
\hline 28. $€ 30.002$ weeks vs. $€ 30.901$ month & 28. $-€ 30.002$ weeks vs. $-€ 30.901$ month \\
\hline 29. $€ 30.002$ weeks vs. $€ 30.901$ month and 2 weeks & 29. $-€ 30.002$ weeks vs. $-€ 30.901$ month and 2 weeks \\
\hline 30. $€ 30.001$ month vs. $€ 30.901$ month and 2 weeks & 30. $-€ 30.001$ month vs. $-€ 30.901$ month and 2 weeks \\
\hline 31. $€ 5.00$ today vs. $€ 5.252$ weeks & 31. $-€ 5.00$ today vs. $-€ 5.252$ weeks \\
\hline 32. $€ 5.00$ today vs. $€ 5.251$ month & 32. $-€ 5.00$ today vs. $-€ 5.251$ month \\
\hline 33. €5.00 2 weeks vs. €5.25 1 month & 33. -€5.00 2 weeks vs. $-€ 5.251$ month \\
\hline 34. $€ 5.002$ weeks vs. $€ 5.251$ month and 2 weeks & 34. $-€ 5.002$ weeks vs. $-€ 5.251$ month and 2 weeks \\
\hline 35. $€ 5.001$ month vs. $€ 5.251$ month and 2 weeks & 35. $-€ 5.001$ month vs. $-€ 5.251$ month and 2 weeks \\
\hline 36. $€ 15.00$ today vs. $€ 15.752$ weeks & 36. $-€ 15.00$ today vs. $-€ 15.752$ weeks \\
\hline 37. $€ 15.00$ today vs. $€ 15.751$ month & 37. $-€ 15.00$ today vs. $-€ 15.751$ month \\
\hline 38. $€ 15.002$ weeks vs. $€ 15.751$ month & 38. $-€ 15.002$ weeks vs. $-€ 15.751$ month \\
\hline 39. $€ 15.002$ weeks vs. $€ 15.751$ month and 2 weeks & 39. $-€ 15.002$ weeks vs. $-€ 15.751$ month and 2 weeks \\
\hline 40. $€ 15.001$ month vs. $€ 15.751$ month and 2 weeks & 40. $-€ 15.001$ month vs. $-€ 15.751$ month and 2 weeks \\
\hline 41. $€ 30.00$ today vs. $€ 31.502$ weeks & 41. $-€ 30.00$ today vs. $-€ 31.502$ weeks \\
\hline 42. $€ 30.00$ today vs. $€ 31.501$ month & 42. $-€ 30.00$ today vs. $-€ 31.501$ month \\
\hline 43. $€ 30.002$ weeks vs. $€ 31.501$ month & 43. $-€ 30.002$ weeks vs. $-€ 31.501$ month \\
\hline 44. $€ 30.002$ weeks vs. $€ 31.501$ month and 2 weeks & 44. $-€ 30.002$ weeks vs. $-€ 31.501$ month and 2 weeks \\
\hline 45. $€ 30.001$ month vs. $€ 31.501$ month and 2 weeks & 45. $-€ 30.001$ month vs. $-€ 31.501$ month and 2 weeks \\
\hline 46. $€ 5.00$ today vs. $€ 5.502$ weeks & 46. $-€ 5.00$ today vs. $-€ 5.502$ weeks \\
\hline 47. $€ 5.00$ today vs. $€ 5.501$ month & 47. $-€ 5.00$ today vs. $-€ 5.501$ month \\
\hline 48. $€ 5.002$ weeks vs. $€ 5.501$ month & 48. $-€ 5.002$ weeks vs. $-€ 5.501$ month \\
\hline 49. $€ 5.002$ weeks vs. $€ 5.501$ month and 2 weeks & 49. $-€ 5.002$ weeks vs. $-€ 5.501$ month and 2 weeks \\
\hline 50. $€ 5.001$ month vs. $€ 5.501$ month and 2 weeks & 50. $-€ 5.001$ month vs. $-€ 5.501$ month and 2 weeks \\
\hline 51. $€ 15.00$ today vs. $€ 16.502$ weeks & 51. $-€ 15.00$ today vs. $-€ 16.502$ weeks \\
\hline
\end{tabular}




\begin{tabular}{|c|c|}
\hline 52. $€ 15.00$ today vs. $€ 16.501$ month & 52. $-€ 15.00$ today vs. $-€ 16.501$ month \\
\hline 53. $€ 15.002$ weeks vs. $€ 16.501$ month & 53. $-€ 15.002$ weeks vs. $-€ 16.501$ month \\
\hline 54. $€ 15.002$ weeks vs. $€ 16.501$ month and 2 weeks & 54. $-€ 15.002$ weeks vs. $-€ 16.501$ month and 2 weeks \\
\hline 55. €15.00 1 month vs. $€ 16.501$ month and 2 weeks & 55. $-€ 15.001$ month vs. $-€ 16.501$ month and 2 weeks \\
\hline 56. $€ 30.00$ today vs. $€ 33.002$ weeks & 56. $-€ 30.00$ today vs. $-€ 33.002$ weeks \\
\hline 57. $€ 30.00$ today vs. $€ 33.001$ month & 57. $-€ 30.00$ today vs. $-€ 33.001$ month \\
\hline 58. $€ 30.002$ weeks vs. $€ 33.001$ month & 58. $-€ 30.002$ weeks vs. $-€ 33.001$ month \\
\hline 59. $€ 30.002$ weeks vs. $€ 33.001$ month and 2 weeks & 59. $-€ 30.002$ weeks vs. $-€ 33.001$ month and 2 weeks \\
\hline 60. $€ 30.001$ month vs. $€ 33.001$ month and 2 weeks & 60. $-€ 30.001$ month vs. $-€ 33.001$ month and 2 weeks \\
\hline 61. $€ 5.00$ today vs. $€ 5.752$ weeks & 61. $-€ 5.00$ today vs. $-€ 5.752$ weeks \\
\hline 62. $€ 5.00$ today vs. $€ 5.751$ month & 62. $-€ 5.00$ today vs. $-€ 5.751$ month \\
\hline 63. $€ 5.002$ weeks vs. $€ 5.751$ month & 63. $-€ 5.002$ weeks vs. $-€ 5.751$ month \\
\hline 64. $€ 5.002$ weeks vs. $€ 5.751$ month and 2 weeks & 64. $-€ 5.002$ weeks vs. $-€ 5.751$ month and 2 weeks \\
\hline 65. €5.00 1 month vs. $€ 15.751$ month and 2 weeks & 65. $-€ 5.001$ month vs. $-€ 15.751$ month and 2 weeks \\
\hline 66. $€ 15.00$ today vs. $€ 17.252$ weeks & 66. $-€ 15.00$ today vs. $-€ 17.252$ weeks \\
\hline 67. $€ 15.00$ today vs. $€ 17.251$ month & 67. $-€ 15.00$ today vs. $-€ 17.251$ month \\
\hline 68. $€ 15.002$ weeks vs. $€ 17.251$ month & 68. $-€ 15.002$ weeks vs. $-€ 17.251$ month \\
\hline 69. $€ 15.002$ weeks vs. $€ 17.251$ month and 2 weeks & 69. $-€ 15.002$ weeks vs. $-€ 17.251$ month and 2 weeks \\
\hline 70. $€ 15.001$ month vs. $€ 17.251$ month and 2 weeks & 70. $-€ 15.001$ month vs. $-€ 17.251$ month and 2 weeks \\
\hline 71. $€ 30.00$ today vs. $€ 34.502$ weeks & 71. $-€ 30.00$ today vs. $-€ 34.502$ weeks \\
\hline 72. $€ 30.00$ today vs. $€ 34.501$ month & 72. $-€ 30.00$ today vs. $-€ 34.501$ month \\
\hline 73. €30.00 2 weeks vs. €34.50 1 month & 73. $-€ 30.002$ weeks vs. $-€ 34.501$ month \\
\hline 74. $€ 30.002$ weeks vs. $€ 34.501$ month and 2 weeks & 74. $-€ 30.002$ weeks vs. $-€ 34.501$ month and 2 weeks \\
\hline 75. $€ 30.001$ month vs. $€ 34.501$ month and 2 weeks & 75. $-€ 30.001$ month vs. $-€ 34.501$ month and 2 weeks \\
\hline 76. $€ 5.00$ today vs. $€ 6.252$ weeks & 76. $-€ 5.00$ today vs. $-€ 6.252$ weeks \\
\hline 77. $€ 5.00$ today vs. $€ 6.251$ month & 77. $-€ 5.00$ today vs. $-€ 6.251$ month \\
\hline 78. $€ 5.002$ weeks vs. $€ 6.251$ month & 78. $-€ 5.002$ weeks vs. $-€ 6.251$ month \\
\hline 79. $€ 5.002$ weeks vs. $€ 6.251$ month and 2 weeks & 79. $-€ 5.002$ weeks vs. $-€ 6.251$ month and 2 weeks \\
\hline 80. $€ 5.001$ month vs. $€ 6.251$ month and 2 weeks & 80. $-€ 5.001$ month vs. $-€ 6.251$ month and 2 weeks \\
\hline 81. $€ 15.00$ today vs. $€ 18.752$ weeks & 81. $-€ 15.00$ today vs. $-€ 18.752$ weeks \\
\hline 82. $€ 15.00$ today vs. $€ 18.751$ month & 82. $-€ 15.00$ today vs. $-€ 18.751$ month \\
\hline 83. $€ 15.002$ weeks vs. $€ 18.751$ month & 83. $-€ 15.002$ weeks vs. $-€ 18.751$ month \\
\hline
\end{tabular}




\begin{tabular}{|c|c|}
\hline 84. $€ 15.002$ weeks vs. $€ 18.751$ month and 2 weeks & 84. $-€ 15.002$ weeks vs. $-€ 18.751$ month and 2 weeks \\
\hline 85. $€ 15.001$ month vs. $€ 18.751$ month and 2 weeks & 85. $-€ 15.001$ month vs. $-€ 18.751$ month and 2 weeks \\
\hline 86. $€ 30.00$ today vs. $€ 37.502$ weeks & 86. $-€ 30.00$ today vs. $-€ 37.502$ weeks \\
\hline 87. $€ 30.00$ today vs. $€ 37.501$ month & 87. $-€ 30.00$ today vs. $-€ 37.501$ month \\
\hline 88. $€ 30.002$ weeks vs. $€ 37.501$ month & 88. $-€ 30.002$ weeks vs. $-€ 37.501$ month \\
\hline 89. $€ 30.002$ weeks vs. $€ 37.501$ month and 2 weeks & 89. $-€ 30.002$ weeks vs. $-€ 37.501$ month and 2 weeks \\
\hline 90. $€ 30.001$ month vs. $€ 37.501$ month and 2 weeks & 90. $-€ 30.001$ month vs. $-€ 37.501$ month and 2 weeks \\
\hline 91. $€ 5.00$ today vs. $€ 6.752$ weeks & 91. $-€ 5.00$ today vs. $-€ 6.752$ weeks \\
\hline 92. $€ 5.00$ today vs. $€ 6.751$ month & 92. $-€ 5.00$ today vs. $-€ 6.751$ month \\
\hline 93. $€ 5.002$ weeks vs. $€ 6.751$ month & 93. $-€ 5.002$ weeks vs. $-€ 6.751$ month \\
\hline 94. $€ 5.002$ weeks vs. $€ 6.751$ month and 2 weeks & 94. $-€ 5.002$ weeks vs. $-€ 6.751$ month and 2 weeks \\
\hline 95. $€ 5.001$ month vs. $€ 6.751$ month and 2 weeks & 95. $-€ 5.001$ month vs. $-€ 6.751$ month and 2 weeks \\
\hline 96. $€ 15.00$ today vs. $€ 20.252$ weeks & 96. $-€ 15.00$ today vs. $-€ 20.252$ weeks \\
\hline 97. $€ 15.00$ today vs. $€ 20.251$ month & 97. $-€ 15.00$ today vs. $-€ 20.251$ month \\
\hline 98. $€ 15.002$ weeks vs. $€ 20.251$ month & 98. $-€ 15.002$ weeks vs. $-€ 20.251$ month \\
\hline 99. $€ 15.002$ weeks vs. $€ 20.251$ month and 2 weeks & 99. $-€ 15.002$ weeks vs. $-€ 20.251$ month and 2 weeks \\
\hline 100.€15.00 1 month vs. $€ 20.251$ month and 2 weeks & 100.- $€ 15.001$ month vs. $-€ 20.251$ month and 2 weeks \\
\hline $101 . € 30.00$ today vs. $€ 40.502$ weeks & 101. $-€ 30.00$ today vs. $-€ 40.502$ weeks \\
\hline $102 . € 30.00$ today vs. $€ 40.501$ month & 102. $-€ 30.00$ today vs. $-€ 40.501$ month \\
\hline 103.€30.00 2 weeks vs. $€ 40.501$ month & 103. $-€ 30.002$ weeks vs. $-€ 40.501$ month \\
\hline 104.€30.00 2 weeks vs. $€ 40.501$ month and 2 weeks & 104.- $€ 30.002$ weeks vs. $-€ 40.501$ month and 2 weeks \\
\hline 105.€30.00 1 month vs. $€ 40.501$ month and 2 weeks & 105.- $€ 30.001$ month vs. $-€ 40.501$ month and 2 weeks \\
\hline 106.€5.00 today vs. $€ 7.502$ weeks & 106. $€ 5.00$ today vs. $-€ 7.502$ weeks \\
\hline 107.€5.00 today vs. $€ 7.501$ month & 107. $-€ 5.00$ today vs. $-€ 7.501$ month \\
\hline 108.€5.00 2 weeks vs. $€ 7.501$ month & 108. $-€ 5.002$ weeks vs. $-€ 7.501$ month \\
\hline 109.€5.00 2 weeks vs. €7.50 1 month and 2 weeks & 109. $€ 5.002$ weeks vs. $-€ 7.501$ month and 2 weeks \\
\hline $110 . € 5.001$ month vs. $€ 7.501$ month and 2 weeks & 110. $-€ 5.001$ month vs. $-€ 7.501$ month and 2 weeks \\
\hline $111 . € 15.00$ today vs. $€ 22.502$ weeks & 111. $-€ 15.00$ today vs. $-€ 22.502$ weeks \\
\hline $112 . € 15.00$ today vs. $€ 22.501$ month & 112. $-€ 15.00$ today vs. $-€ 22.501 \mathrm{month}$ \\
\hline 113.€15.00 2 weeks vs. $€ 22.501$ month & 113. $-€ 15.002$ weeks vs. $-€ 22.501$ month \\
\hline 114.€15.00 2 weeks vs. $€ 22.501$ month and 2 weeks & 114.- $€ 15.002$ weeks vs. $-€ 22.501$ month and 2 weeks \\
\hline $115 . € 15.001$ month vs. $€ 22.501$ month and 2 weeks & 115. $-€ 15.001$ month vs. $-€ 22.501$ month and 2 weeks \\
\hline
\end{tabular}




\begin{tabular}{|l|l|}
\hline $116 . € 30.00$ today vs. $€ 45.002$ weeks & $116 .-€ 30.00$ today vs. $-€ 45.002$ weeks \\
$117 . € 30.00$ today vs. $€ 45.001$ month & $117 .-€ 30.00$ today vs. $-€ 45.001$ month \\
$118 . € 30.002$ weeks vs. $€ 45.001$ month & $118 .-€ 30.002$ weeks vs. $-€ 45.001$ month \\
$119 . € 30.002$ weeks vs. $€ 45.001$ month and 2 weeks & $119 .-€ 30.002$ weeks vs. $-€ 45.001$ month and 2 weeks \\
$120 . € 30.001$ month vs. $€ 45.001$ month and 2 weeks & $120 .-€ 30.001$ month vs. $-€ 45.001$ month and 2 weeks \\
\hline
\end{tabular}




\section{REFERENCES}

Agresti, A. (2002). Categorical Data Analysis (2 ${ }^{\text {nd }}$ edition ed.). New Jersey: Wiley.

Benzion, U., Rapoport, A., \& Yagil, J. (1989). Discount rates inferred from decisions: an experimental study. Management Science, 35(3), 270-284.

Bickel, W. K., \& Madden, G. J. (1999). A comparison of measures of relative reinforcing efficacy and behavioral economics: cigarettes and money in smokers. Behavioural Pharmacology, 10(6-7), 627-637.

Cook, J. O., \& Barnes, L. W., Jr. (1964). Choice of Delay of Inevitable Shock. Journal of Abnormal Psychology, 68, 669-672.

Croson, R., \& Gneezy, U. (2009). Gender differences in preferences. Journal of Economic Literature, 47(2), 448-474.

Dimitri, N. (2007). A note on the reward-loss duality in time consistent decisions. University of Siena, Department of Economic Policy, Finance and Development, Working paper 8.

Fisher, I. (1930). The theory of interest. New York: MacMillan.

Frederick, S., Loewenstein, G., \& O’Donoghue, T. (2002). Time discounting and time preference: a critical review. Journal of Economic Literature, 40(2), 351-401.

Grusec, J. E. (1968). Waiting for rewards and punishments: effects of reinforcement value on choice. Journal of Personality and Social Psychology, 9(1), 85-89.

Hare, R. D. (1966). Preference for delay of shock as a function of its intensity and probability. Psychonomic Science, 5, 393-394.

Hare, R. D., Krebs, D. L., Creighton, T. D., \& Petrusic, W. M. (1966). Latency of self-administered shock as a function of its intensity and probability. Psychonomic Science, 6, 79-80.

Kirby, K. N., Petry, N. M., \& Bickel, W. K. (1999). Heroin addicts have higher discount rates for delayed rewards than non-drug-using controls. Journal of Experimental Psychology: General, $128(1), 78-87$. 
Loewenstein, G. (1987). Anticipation and the valuation of delayed consumption. The Economic Journal, 97(387), 666-684.

Loewenstein, G. (1988). The weighting of waiting: response mode effects in intertemporal choice. University of Chicago, Center for Decision Research, Working paper.

Loewenstein, G., \& Prelec, D. (1992). Anomalies in intertemporal choice: evidence and interpretation. The Quarterly Journal of Economics, 107(2), 573-597.

Loewenstein, G., \& Sicherman, N. (1991). Do worker prefer increasing wage profiles? Journal of Labor Economics, 9(1), 67-84.

Madden, G. J., Petry, N. M., Badger, G. J., \& Bickel, W. K. (1997). Impulsive and self-control choices in opioid-dependent patients and non-drug-using control participants: drug and monetary rewards. Experimental and Clinical Psychopharmacology, 5(3), 256-262.

McClure, S. M., Laibson, D. I., Loewenstein, G., \& Cohen, J. D. (2004). Separate neural systems value immediate and delayed monetary rewards. Science, 306(5695), 503-507.

Mischel, W., Grusec, J., \& Masters, J. C. (1969). Effects of expected delay time on the subjective value of rewards and punishments. Journal of Personality and Social Psychology 11(4), 363-373.

relec, D., \& Loewenstein, G. (1998). The red and the black: mental accounting of savings and debt. Marketing Science, 17(1), 4-28.

Rae, J. (1834). The social theory of capital. London: MacMillan.

Read, D. (2004). Intertemporal choice. In D. Koehler \& N. Harvey (Eds.), Blackwell Handbook of Judgment and Decision Making (pp. 424-443). Oxford: Blackwell.

Samuelson, P. A. (1937). A note on measurement of utility. Review of Economic Studies, 4(2), 155161.

Shelley, M. K. (1993). Outcome signs, question frames and discount rates. Management Science, 39(7), 806-815.

Strotz, R. (1955-56). Myopia and inconsistency in dynamic utility maximization. Review of Economic Studies, 23(3), 165-180. 
Thaler, R. H. (1981). Some empirical evidence on dynamic inconsistency. Economic Letters, 8, 201-207.

Xu, L., Liang, Z. Y., Wang, K., Li, S., \& Jiang, T. (2009). Neural mechanism of intertemporal choice: from discounting future gains to future losses. Brain Research, 1261, 65-74.

Yates, J. F., \& Watts, R. A. (1975). Preferences for deferred losses. Organizational Behavior and Human Performances, 13(2), 294-306. 